\title{
Generalized Degrees of Freedom of the (Noisy) $X$ Channel
}

\author{
Chiachi Huang, Viveck Cadambe, Syed A. Jafar \\ Electrical Engineering and Computer Science \\ University of California Irvine \\ Irvine, CA, USA \\ Email: $\{$ chiachih, vcadame, syed $\}$ uci.edu
}

\begin{abstract}
We provide outer bounds on the capacity region of the two-user Gaussian $X$ channel, i.e. a generalization of the twouser interference channel where there is an independent message from each transmitter to each receiver. We identify the conditions under which operating the $X$ channel as the interference channel is optimal from the perspective of generalized degrees of freedom (GDOF) and sum capacity. Specifically, we first extend the bound on the sum rate of the interference channel obtained by Etkin, Tse, and Wang in [1] to the $X$ channel. This bound provides insights into the operating regimes in which two channels have the same GDOF. We then extend the noisy interference capacity characterization previously obtained for the interference channel [2]-[4] to the $X$ channel. Therefore, we show that the $X$ channel associated with noisy (very weak) interference channel has the same sum capacity as the noisy interference channel.
\end{abstract}

\section{INTRODUCTION}

Interference is the key property of wireless communication due to the broadcasting nature of wireless links. The model that is widely used to study the behavior and the corresponding management of interference is the interference channel. Unfortunately, the characterization of the capacity region of the interference channel has been an open problem over thirty years. Recently, significant progress is made by Etkin, Tse, and Wang [1] to approximate the capacity region of the two-user Gaussian interference channel to within one bit. Further insight into the capacity of the the channel is revealed in [2]-[4]. These references find that the decoding strategy of treating interference as noise at each receiver in the interference network is capacity optimal for a class of interference channels, known as the "noisy" interference channels. Recent results have also found approximations to the capacity regions of certain $K$-user interference channels in the high signal-to-noise ratio (SNR) regime. Reference [5] approximates the capacity region of the fully connected $K$-user interference channel with time-varying channel coefficients as

$$
C(\mathrm{SNR})=\frac{K}{2} \log (\mathrm{SNR})+o(\log (\mathrm{SNR}))
$$

where SNR represents the total transmit power of all nodes when the local noise power at each receiver is normalized to unity. In other words, it was shown that the time-varying $K$ user interference channel has $\frac{K}{2}$ degrees of freedom. Similar capacity approximations of the $K$-user $(K>2)$ interference channel with constant channel coefficients (i.e., not timevarying or frequency-selective) are not known in general.
From the recent advances in the study of interference channels, many interesting and powerful tools related to the study of general wireless networks have emerged. Reference [1] introduces the notion of generalized degrees of freedom (GDOF) to study the performance of various interference management schemes. As its name suggests, the idea of GDOF is a generalization of the concept of degrees of freedom originally introduced in [6]. Unlike the conventional degrees of freedom perspective where all signals are approximately equally strong in the $d B$ scale, the GDOF perspective provides a richer characterization by allowing the full range of relative signal strengths in the $d B$ scale. The idea of GDOF is powerful because in the multiple access, broadcast and twouser interference channels, achievable schemes that are optimal from a GDOF perspective also achieve within a constant number of bits of capacity [7].

In this paper, we explore the two-user $X$ channel - a network with two transmitters, two receivers and four independent messages - one corresponding to each transmitter-receiver pair. One of the key features of the $X$ channel is that, unlike the two-user interference channel, it provides the possibility of interference alignment [8] [9]. Interference alignment refers to the construction of signals such that they overlap at receivers where they cause interference, but remain distinguishable at receivers where they are desired. Interference alignment is the key to the degrees of freedom characterizations of the $X$ channel with two or more users [10], and for the interference channel with three or more users [5]. Since the potential for interference alignment does not arise in the two-user interference channel, the two-user $X$ channel provides the simplest possible setting for interference alignment, in terms of the number of transmitters/receivers and channel coefficients. It is shown in [8] that, due to interference alignment, the two-user $X$ channel has $4 / 3$ degrees of freedom (assuming time-varying channels), while the two-user interference channel has only 1 degree of freedom. In this paper, instead of trying to apply interference alignment to find a larger achievable region, we take an alternative path and try to answer the following questions. When don't extra messages help? When doesn't interference alignment work? We answer these questions by identifying the conditions under which operating the $X$ channel as the interference channel is optimal from the perspective of GDOF and capacity respectively. 
The rest of the paper is organized as follows. Section II describes the model. In Section III, we provide the main results of the paper. The detailed derivations of our GDOF and capacity results are given in Section IV and V respectively. Section VI concludes the paper.

\section{SySTEM MOdEL}

The two-user Gaussian $X$ channel is described by the inputoutput equations

$$
\begin{aligned}
& Y_{1}(t)=H_{11} X_{1}(t)+H_{12} X_{2}(t)+Z_{1}(t) \\
& Y_{2}(t)=H_{21} X_{1}(t)+H_{22} X_{2}(t)+Z_{2}(t)
\end{aligned}
$$

where at symbol index $t, Y_{j}(t)$ and $Z_{j}(t)$ are the channel output symbol and additive white Gaussian noise (AWGN) respectively at receiver $j . X_{i}(t)$ is the channel input symbol at transmitter $i$, and $H_{j i}$ is the channel gain coefficient between transmitter $i$ and receiver $j$ for all $i, j \in\{1,2\}$. All symbols are real and the channel coefficients do not vary w.r.t symbol index. In the remainder of this paper, we suppress time index $t$ if no confusion would be caused. The AWGN is normalized to have zero mean and unit variance and the input power constraint is given by

$$
\mathbf{E}\left[X_{i}^{2}\right] \leq P_{i}, \quad i=1,2 .
$$

There are four independent messages in the $X$ channel: $W_{11}, W_{12}, W_{21}, W_{22}$ where $W_{j i}$ represents the message from transmitter $j$ to receiver $i$. We indicate the size of the message by $\left|W_{j i}\right|$. For codewords spanning $T$ symbols, rates $R_{i j}=\frac{\log \left|W_{j i}\right|}{T}$ are achievable if the probability of error for all messages can be simultaneously made arbitrarily small by choosing an appropriate large $T$. The capacity region $\mathcal{C}$ of the $X$ channel is the set of all achievable rate tuples $\mathbf{R}=\left(R_{11}, R_{12}, R_{21}, R_{22}\right)$. We indicate the sum capacity of the $X$ channel by $C_{\Sigma}$.

\section{A. Generalized Degrees of Freedom (GDOF)}

To motivate our problem formulation, we briefly revisit the framework for the GDOF characterization of the symmetric interference channel. The interference channel is defined as:

$$
\begin{aligned}
& Y_{1}(t)=\sqrt{\mathrm{SNR}} X_{1}(t)+\sqrt{\mathrm{INR}} X_{2}(t)+Z_{1}(t) \\
& Y_{2}(t)=\sqrt{\mathrm{INR}} X_{1}(t)+\sqrt{\mathrm{SNR}} X_{2}(t)+Z_{2}(t)
\end{aligned}
$$

and with the parameter $\alpha$ defined as follows

$$
\alpha \triangleq \frac{\log (\mathrm{INR})}{\log (\mathrm{SNR})}
$$

the GDOF metric is defined as [1],

$$
d(\alpha)=\limsup _{\mathrm{SNR} \rightarrow \infty} \frac{C_{\Sigma}(\mathrm{SNR}, \alpha)}{\frac{1}{2} \log (\mathrm{SNR})}
$$

where $C_{\Sigma}(\mathrm{SNR}, \alpha)$ is the sum capacity of the interference channel.

Since our main goal is to compare GDOF of the $X$ channel with the interference channel, we use the same symmetric interference channel model described above as the physical

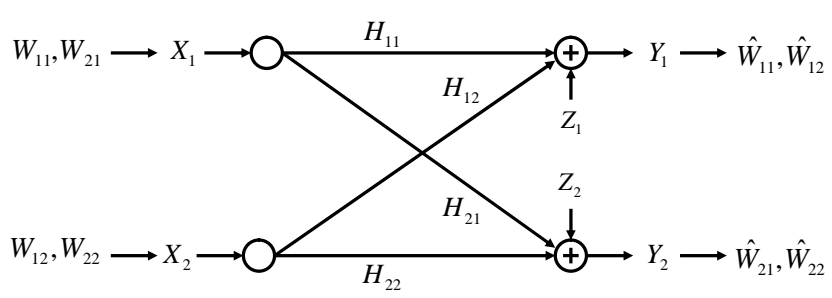

Fig. 1. Two-user Gaussian $X$ channel.

channel model for the $X$ channel. There is however, one notational difference. Since the terminology SNR, INR is not as appropriate for the $X$ channel, we instead use the parameter $\rho$ to substitute for these notions, resulting in the following system model for the $X$ channel GDOF characterization:

$$
\begin{aligned}
& Y_{1}(t)=\sqrt{\rho} X_{1}(t)+\sqrt{\rho^{\alpha}} X_{2}(t)+Z_{1}(t) \\
& Y_{2}(t)=\sqrt{\rho^{\alpha}} X_{1}(t)+\sqrt{\rho} X_{2}(t)+Z_{2}(t)
\end{aligned}
$$

In other words, we have set $H_{11}=H_{22}=\sqrt{\rho}, H_{12}=$ $H_{21}=\sqrt{\rho^{\alpha}}$, and $P_{1}=P_{2}=1$. Note that (8), (9) represent the same physical channel as (4), (5). However, as mentioned earlier, unlike the interference channel the $X$ channel has 4 independent messages - one from each transmitter to each receiver. The GDOF characterization for the $X$ channel is defined as:

$$
d(\alpha)=\limsup _{\rho \rightarrow \infty} \frac{C_{\Sigma}(\rho, \alpha)}{\frac{1}{2} \log (\rho)}
$$

where $C_{\Sigma}(\rho, \alpha)$ is the sum capacity of the $X$ channel.

Note that we use limsup to ensure that $d(\alpha)$ always exits. The half in the denominator is because all signals and channel gains are real.

We use the following notation convention. $A^{(T)}$ is defined as

$$
A^{(T)} \triangleq(A(1), A(2), \ldots A(T)),
$$

for any sequence $A$. Similar to the notation usage in [4], we use $X_{i G}$ to denote zero-mean Gaussian random variables with variance $P_{i}$ for $i=1,2$. A subscript $G$ is added to a random variable when all its associated channel inputs are Gaussian.

\section{Main Results}

\section{A. GDOF of the Symmetric Gaussian X Channel}

The first main result of this paper is the GDOF characterization of the Gaussian $X$ channel for $\alpha \leq \frac{2}{3}$ and $\alpha>\frac{3}{2}$. For comparison, Fig. 2 also shows the GDOF characterization of the symmetric interference channel as obtained in [1]. For values of $\alpha \leq 2 / 3$, characterization of $d(\alpha)$ is identical for both channels. We prove this by showing that the EtkinTse-Wang (ETW) outerbound derived for the interference channel [1] holds for the $X$ channel as well (See Theorem 1). The ETW outerbound is tight from a GDOF perspective in the interference channel for $\alpha \leq 2 / 3$. Therefore, our 
extension of this outerbound implies that for $\alpha \leq 2 / 3$ a GDOF optimal achievable scheme is to set $W_{12}=W_{21}=\phi$, so that the $X$ channel operates as an interference channel. Similarly, we show that for $\alpha>\frac{3}{2}$, it is GDOF optimal to set $W_{22}=W_{11}=\phi$ and operate the $X$ channel as an interference channel with messages $W_{12}$ and $W_{21}$. Note that for both $\alpha \leq 2 / 3$ and $\alpha>3 / 2$ the GDOF optimal achievable scheme operates the $X$ channel as weak interference channel by setting the appropriate messages to null.

\section{B. Capacity of the "Noisy" Gaussian X Channel}

References [2]-[4] show that in the interference channel, for a class of channel coefficients, encoding messages using Gaussian codebooks and decoding desired messages by treating interference as noise at each receiver is capacity optimal. Our second main result extends this conclusion to the $X$ channel. We show that if a two-user interference channel satisfies the noisy interference conditions obtained in [2]-[4] then the corresponding $X$ channel obtained by allowing all transmitters to communicate with all receivers, has the same sum capacity as the original noisy interference channel. This is a surprising result since it implies that for a class of $X$ channels, interference alignment has no capacity benefit. For simplicity we re-state the result here for the symmetric case $\left(H_{11}=H_{22}=1, H_{12}=H_{21}=h, P_{1}=P_{2}=P\right)$ in a notation consistent with [4], as follows.

Noisy "Symmetric" X Channel Result: If $\left|h\left(1+h^{2} P\right)\right| \leq$ $\frac{1}{2}$, then the sum capacity of the Gaussian $X$ channel is given by $C_{\Sigma}=\log \left(1+\frac{P}{1+h^{2} P}\right)$. Similarly, if $|h| \geq 2(1+P)$ then the sum capacity of the Gaussian $X$ channel is given by $C_{\Sigma}=$ $\log \left(1+\frac{h^{2} P}{1+P}\right)$.

The condition $\left|h\left(1+h^{2} P\right)\right| \leq \frac{1}{2}$ is the same as the noisy interference condition in [4]. It means that when the cross-links are too weak, there is no sum-capacity benefit in communicating messages over those links ( $X$ channel operation), even though it rules out interference alignment, and we are better off just communicating on the direct links while treating the weak interference as noise. Thus, in this case messages $W_{12}, W_{21}$ do not increase sum capacity of the $X$ channel.

The other condition $|h| \geq 2(1+P)$ refers to a strong crosschannel scenario. It says that when the cross-links are too strong relative to direct links, then sum capacity is achieved by communicating only over the strong cross-links and treating the weak interference received over the direct links as noise. In this case, messages $W_{11}, W_{22}$ do not increase the sum capacity of the $X$ channel.

\section{GDOF of the Symmetric Gaussian $X$ Channel}

In this section, we extend the ETW outerbound derived for the interference channel to the $X$ channel, and use it to find the generalized degrees of freedom for $X$ channel for $\alpha \leq \frac{2}{3}$ and $\alpha>\frac{3}{2}$. We start from the following theorem.

Theorem 1: The sum rate $R_{\Sigma}$ achieved by any reliable coding scheme over the $X$ channel satisfies the following

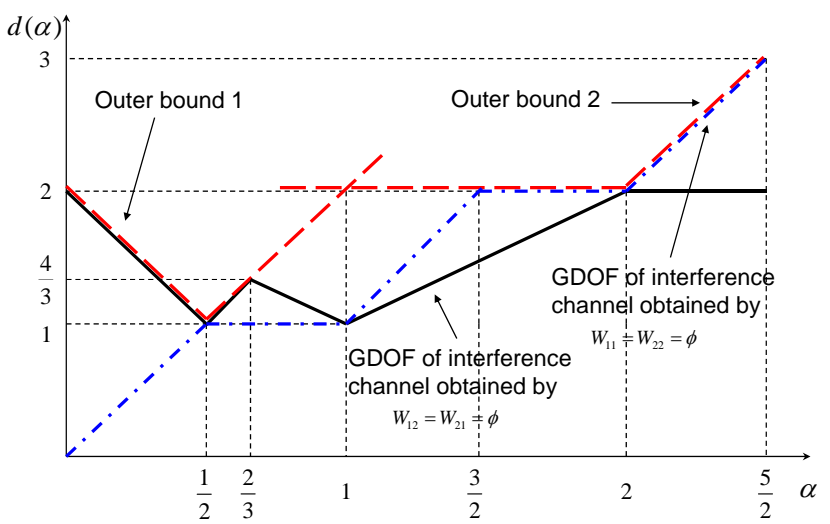

Fig. 2. Outer and inner bounds for the generalized degrees of freedom of the two-user Gaussian $X$ channel.

bounds

$$
\begin{aligned}
& R_{\Sigma} \leq \frac{1}{2} \log \left(1+H_{12}{ }^{2} P_{2}+\frac{H_{11}{ }^{2} P_{1}}{1+{H_{21}}^{2} P_{1}}\right) \\
&+\frac{1}{2} \log \left(1+H_{21}{ }^{2} P_{1}+\frac{H_{22}{ }^{2} P_{2}}{1+H_{12}{ }^{2} P_{2}}\right) \\
& R_{\Sigma} \leq \frac{1}{2} \log \left(1+H_{11}{ }^{2} P_{1}+\frac{H_{12}{ }^{2} P_{2}}{1+H_{22}{ }^{2} P_{2}}\right) \\
&+\frac{1}{2} \log \left(1+H_{22}{ }^{2} P_{2}+\frac{H_{21}{ }^{2} P_{1}}{1+H_{11}{ }^{2} P_{1}}\right)
\end{aligned}
$$

Proof: Let

$$
\begin{aligned}
& S_{1}(t)=H_{21} X_{1}(t)+Z_{2}(t) \\
& S_{2}(t)=H_{12} X_{2}(t)+Z_{1}(t) .
\end{aligned}
$$

Note that $S_{1}$ and $S_{2}$ are auxiliary variables similar to those used in the ETW outerbound of the interference channel. Consider any reliable coding scheme. Now, let a genie provide $S_{2}$ and $W_{12}$ to receiver 2. From Fano's inequality, for any codeword of length $T$, we can write

$$
\begin{aligned}
T( & \left.R_{22}+R_{21}-\epsilon\right) \\
\leq & I\left(W_{22}, W_{21} ; Y_{2}^{(T)}, S_{2}^{(T)}, W_{12}\right) \\
\stackrel{(a)}{=} & I\left(W_{22}, W_{21} ; Y_{2}^{(T)}, S_{2}^{(T)} \mid W_{12}\right) \\
= & I\left(W_{22}, W_{21} ; S_{2}^{(T)} \mid W_{12}\right)+I\left(W_{22}, W_{21} ; Y_{2}^{(T)} \mid W_{12}, S_{2}^{(T)}\right) \\
= & h\left(S_{2}^{(T)} \mid W_{12}\right)-h\left(S_{2}^{(T)} \mid W_{12}, W_{22}, W_{21}\right) \\
& +h\left(Y_{2}^{(T)} \mid W_{12}, S_{2}^{(T)}\right)-h\left(Y_{2}^{(T)} \mid W_{12}, S_{2}^{(T)}, W_{22}, W_{21}\right) \\
\stackrel{(b)}{\leq} & h\left(S_{2}^{(T)} \mid W_{12}\right)-h\left(S_{2}^{(T)} \mid W_{12}, W_{22}, W_{21}, X_{2}^{(T)}\right) \\
& +h\left(Y_{2}^{(T)} \mid S_{2}^{(T)}\right)-h\left(Y_{2}^{(T)} \mid W_{12}, S_{2}^{(T)}, W_{22}, W_{21}, X_{2}^{(T)}\right) \\
= & h\left(S_{2}^{(T)} \mid W_{12}\right)-h\left(Z_{1}^{(T)} \mid W_{12}, W_{22}, W_{21}, X_{2}^{(T)}\right) \\
& +h\left(Y_{2}^{(T)} \mid S_{2}^{(T)}\right)-h\left(S_{1}^{(T)} \mid W_{12}, S_{2}^{(T)}, W_{22}, W_{21}, X_{2}^{(T)}\right) \\
= & h\left(S_{2}^{(T)} \mid W_{12}\right)-h\left(Z_{1}^{(T)}\right)+h\left(Y_{2}^{(T)} \mid S_{2}^{(T)}\right)-h\left(S_{1}^{(T)} \mid W_{21}\right) \\
&
\end{aligned}
$$


where in step (a) we use the fact all messages are independent, and in step (b) we use the fact that $X_{2}^{(T)}$ is a function of $W_{12}$ and $W_{22}$ alone with the fact that dropping conditioning does not reduce differential entropy.

Similarly, by letting a genie provide receiver 1 with $S_{1}^{(T)}$ and $W_{21}$, we can bound the rates at receiver 1 as

$$
\begin{aligned}
& T\left(R_{11}+R_{12}-\epsilon\right) \\
& \quad \leq h\left(S_{1}^{(T)} \mid W_{21}\right)-h\left(Z_{2}^{(T)}\right)+h\left(Y_{1}^{(T)} \mid S_{1}^{(T)}\right)-h\left(S_{2}^{(T)} \mid W_{12}\right) .
\end{aligned}
$$

Adding (15) and (16), we get

$$
\begin{aligned}
& T\left(R_{\Sigma}-\epsilon\right) \\
& \leq h\left(Y_{1}^{(T)} \mid S_{1}^{(T)}\right)+h\left(Y_{2}^{(T)} \mid S_{2}^{(T)}\right)-h\left(Z_{1}^{(T)}\right)-h\left(Z_{2}^{(T)}\right) \\
& \leq \sum_{t=1}^{T}\left[h\left(Y_{1}(t) \mid S_{1}(t)\right)+h\left(Y_{2}(t) \mid S_{2}(t)\right)\right]-T h\left(Z_{1}\right)-T h\left(Z_{2}\right)
\end{aligned}
$$

where the second inequality follows by fact that dropping conditioning does not reduce differential entropy. Dividing both sides by $T$, taking $T \rightarrow \infty$, and using the fact that independent and identical (i.i.d.) Gaussian random variables maximize conditional differential entropy for a given covariance constraint, we get the desired outer bound (11).

Similarly, the second bound on the sum capacity of the $X$ channel can be proved by letting a genie provide $H_{22} X_{2}^{(T)}+$ $Z_{2}^{(T)}$ and $W_{22}$ to receiver 1 and $H_{11} X_{1}^{(T)}+Z_{1}^{(T)}$ and $W_{11}$ to receiver 2 . This completes the proof.

The following theorem gives the characterization of $d(\alpha)$ for $\alpha \leq \frac{2}{3}$ and $\alpha>\frac{3}{2}$.

Theorem 2:

$$
\begin{array}{ll}
d(\alpha)=2 \max (\alpha, 1-\alpha), & \text { for } \alpha \leq \frac{2}{3} \\
d(\alpha)=2 \max (1, \alpha-1), & \text { for } \alpha>\frac{3}{2}
\end{array}
$$

Proof: Substituting $H_{11}=H_{22}=\sqrt{\rho}, H_{21}=H_{12}=$ $\sqrt{\rho^{\alpha}}$, and $P_{1}=P_{2}=1$ into (11), dividing both sides by $\frac{1}{2} \log \rho$, and letting $\rho \rightarrow \infty$, we get

$$
d(\alpha) \leq 2 \max (\alpha, 1-\alpha) .
$$

Following the same steps except that now we substitute $H_{21}=$ $H_{12}=\sqrt{\rho}$ and $H_{11}=H_{22}=\sqrt{\rho^{\alpha}}$, and $P_{1}=P_{2}=1$ into (12), we get

$$
d(\alpha) \leq 2 \max (1, \alpha-1) .
$$

By comparing the outer and inner bounds shown in Fig. 2, we finish the proof.

\section{Capacity of the "Noisy" Gaussian $X$ Channel}

\section{Theorem 3: If}

$$
\left|\frac{H_{12}}{H_{22}}\left(1+H_{21}^{2} P_{1}\right)\right|+\left|\frac{H_{21}}{H_{11}}\left(1+H_{12}^{2} P_{2}\right)\right| \leq 1,
$$

then the sum capacity of the Gaussian $X$ channel is given by

$$
C_{\Sigma}=\frac{1}{2} \log \left(1+\frac{H_{11}^{2} P_{1}}{1+H_{12}^{2} P_{2}}\right)+\frac{1}{2} \log \left(1+\frac{H_{22}^{2} P_{2}}{1+H_{21}^{2} P_{1}}\right) \text {. }
$$

Similarly, if

$$
\left|\frac{H_{22}}{H_{12}}\left(1+H_{11}^{2} P_{1}\right)\right|+\left|\frac{H_{11}}{H_{21}}\left(1+H_{22}^{2} P_{2}\right)\right| \leq 1,
$$

then the sum capacity of the Gaussian $X$ channel is given by

$$
C_{\Sigma}=\frac{1}{2} \log \left(1+\frac{H_{21}^{2} P_{1}}{1+H_{22}^{2} P_{2}}\right)+\frac{1}{2} \log \left(1+\frac{H_{12}^{2} P_{2}}{1+H_{11}^{2} P_{1}}\right) \text {. }
$$

Proof: Let

$$
\begin{aligned}
& \tilde{S}_{1}(t)=H_{21}\left(X_{1}(t)+\tilde{Z}_{1}(t)\right) \\
& \tilde{S}_{2}(t)=H_{12}\left(X_{2}(t)+\tilde{Z}_{2}(t)\right)
\end{aligned}
$$

where $\tilde{Z}_{i}$ is white Gaussian with zero mean and variance $\sigma_{i}^{2}$ for $i=1,2$. Also, let $\tilde{Z}_{i}(t)$ be correlated with $Z_{i}(t)$ as

$$
E\left[Z_{i}(t) \tilde{Z}_{i}(t)\right]=\sigma_{i} \rho_{i}, \quad i=1,2
$$

Note that since the variance of $Z_{i}$ is one, $\rho_{i}$ is the correlation coefficient between $Z_{i}$ and $\tilde{Z}_{i}$. Let a genie provide $\tilde{S}_{1}$ and $W_{21}$ to receiver 1 and $\tilde{S}_{2}$ and $W_{12}$ to receiver 2 . Using Fano's inequality for a codeword spanning $T$ symbols, we have

$$
\begin{aligned}
T( & \left.R_{22}+R_{21}-\epsilon\right) \\
\leq & I\left(W_{22}, W_{21} ; Y_{2}^{(T)}, \tilde{S}_{2}^{(T)}, W_{12}\right) \\
\stackrel{(a)}{=} & I\left(W_{22}, W_{21} ; Y_{2}^{(T)}, \tilde{S}_{2}^{(T)} \mid W_{12}\right) \\
= & I\left(W_{22}, W_{21} ; \tilde{S}_{2}^{(T)} \mid W_{12}\right) \\
& +I\left(W_{22}, W_{21} ; Y_{2}^{(T)} \mid W_{12}, \tilde{S}_{2}^{(T)}\right) \\
= & h\left(\tilde{S}_{2}^{(T)} \mid W_{12}\right)-h\left(\tilde{S}_{2}^{(T)} \mid W_{12}, W_{22}, W_{21}\right) \\
& +h\left(Y_{2}^{(T)} \mid W_{12}, \tilde{S}_{2}^{(T)}\right)-h\left(Y_{2}^{(T)} \mid W_{12}, \tilde{S}_{2}^{(T)}, W_{22}, W_{21}\right) \\
= & h\left(\tilde{S}_{2}^{(T)} \mid W_{12}\right)-T h\left(\tilde{Z}_{2}\right)+h\left(Y_{2}^{(T)} \mid W_{12}, \tilde{S}_{2}^{(T)}\right) \\
& -h\left(Y_{2}^{(T)} \mid W_{12}, \tilde{S}_{2}^{(T)}, W_{22}, W_{21}\right) \\
(b) & h\left(\tilde{S}_{2}^{(T)} \mid W_{12}\right)-T h\left(\tilde{Z}_{2}\right)+h\left(Y_{2}^{(T)} \mid \tilde{S}_{2}^{(T)}\right) \\
& -h\left(H_{21} X_{1}^{(T)}+Z_{2}^{(T)} \mid \tilde{Z}_{2}^{(T)}, W_{21}\right)
\end{aligned}
$$

where in step (a) we use the fact that all messages are independent. Step (b) follows by the fact that dropping conditioning does not reduce differential entropy and the fact that $h\left(Y_{2}^{(T)} \mid W_{12}, \tilde{S}_{2}^{(T)}, W_{22}, W_{21}\right)$ can be simplified as the following.

$$
\begin{aligned}
h\left(Y_{2}^{(T)} \mid W_{12}, \tilde{S}_{2}^{(T)}, W_{22}, W_{21}\right) \\
\quad=h\left(Y_{2}^{(T)} \mid W_{12}, \tilde{S}_{2}^{(T)}, W_{22}, W_{21}, X_{2}^{(T)}\right) \\
\quad=h\left(H_{21} X_{1}^{(T)}+Z_{2}^{(T)} \mid W_{12}, \tilde{Z}_{2}^{(T)}, W_{22}, W_{21}, X_{2}^{(T)}\right) \\
\quad=h\left(H_{21} X_{1}^{(T)}+Z_{2}^{(T)} \mid \tilde{Z}_{2}^{(T)}, W_{21}\right)
\end{aligned}
$$

Similarly, we can bound $R_{12}$ and $R_{11}$ as

$$
\begin{aligned}
T\left(R_{12}+R_{11}-\epsilon\right) \leq & h\left(\tilde{S}_{1}^{(T)} \mid W_{21}\right)-T h\left(\tilde{Z}_{1}\right)+h\left(Y_{1}^{(T)} \mid \tilde{S}_{1}^{(T)}\right) \\
& -h\left(H_{12} X_{2}^{(T)}+Z_{1}^{(T)} \mid \tilde{Z}_{1}^{(T)}, W_{12}\right)
\end{aligned}
$$


Adding (26) and (27), we get

$$
\begin{aligned}
T( & \left.R_{\Sigma}-\epsilon\right) \\
\leq & \underbrace{h\left(H_{12} X_{2}^{(T)}+H_{12} \tilde{Z}_{2}^{(T)} \mid W_{12}\right)-h\left(H_{12} X_{2}^{(T)}+Z_{1}^{(T)} \mid \tilde{Z}_{1}^{(T)}, W_{12}\right)}_{U_{1}} \\
& +\underbrace{h\left(H_{21} X_{1}^{(T)}+H_{21} \tilde{Z}_{1}^{(T)} \mid W_{21}\right)-h\left(H_{21} X_{1}^{(T)}+Z_{2}^{(T)} \mid \tilde{Z}_{2}^{(T)}, W_{21}\right)}_{U_{2}} \\
& +\underbrace{h\left(Y_{1}^{(T)} \mid \tilde{S}_{1}^{(T)}\right)+h\left(Y_{2}^{(T)} \mid \tilde{S}_{2}^{(T)}\right)-T h\left(\tilde{Z}_{1}\right)-T h\left(\tilde{Z}_{2}\right)}_{U_{3}}
\end{aligned}
$$

The rest of the proof goes along the similar lines as described in [4]. We only highlight the differences here.

Note that $U_{3}$ is maximized if we choose $X_{1}(t)$ to be i.i.d. Gaussian of variance $P_{1}$, since $h\left(Y_{i}^{(T)} \mid \tilde{S}_{i}^{(T)}\right)$ is maximized by this choice. Thus, we have

$$
U_{3} \leq T h\left(Y_{1 G} \mid \tilde{S}_{1 G}\right)+T h\left(Y_{2 G} \mid \tilde{S}_{2 G}\right)-T h\left(\tilde{Z}_{1}\right)-T h\left(\tilde{Z}_{2}\right)
$$

where $X_{i G}, Y_{i G}$, and $\tilde{S}_{i G}$ are variables obtained by letting $X_{i}$ be a Gaussian random variable of variance $P_{i}$ for $i=1,2$. Now, following the proof of [4], we derive conditions on $\rho_{i}, \sigma_{i}$ for $i=1,2$ so that independent and identical Gaussian distributions on $X_{i}(t)$ maximizes $U_{1}$ and $U_{2}$ as well. Specifically, we show that if

$$
1-\rho_{2}^{2} \geq H_{21}^{2} \sigma_{1}^{2},
$$

then $U_{2}$ can be bounded above as the following.

$$
\begin{aligned}
U_{2} \stackrel{(a)}{=} & h\left(H_{21} X_{1}^{(T)}+H_{21} \tilde{Z}_{1}^{(T)} \mid W_{21}\right) \\
& -h\left(H_{21} X_{1}^{(T)}+V_{1}^{(T)} \mid W_{21}\right) \\
\stackrel{(b)}{=} & h\left(H_{21} X_{1}^{(T)}+H_{21} \tilde{Z}_{1}^{(T)} \mid W_{21}\right) \\
& -h\left(H_{21} X_{1}^{(T)}+H_{21} \tilde{Z}_{1}^{(T)}+V^{(T)} \mid W_{21}\right) \\
= & -I\left(V^{(T)} ; H_{21} X_{1}^{(T)}+H_{21} \tilde{Z}_{1}^{(T)}+V^{(T)} \mid W_{21}\right) \\
\stackrel{(c)}{\leq} & -I\left(V^{(T)} ; H_{21} X_{1}^{(T)}+H_{21} \tilde{Z}_{1}^{(T)}+V^{(T)}\right) \\
\stackrel{(d)}{\leq} & -T I\left(V ; H_{21} X_{1}+H_{21} \tilde{Z}_{1}+V\right) \\
= & T h\left(H_{21} X_{1 G}+H_{21} \tilde{Z}_{1}\right)-T h\left(H_{21} X_{1 G}+Z_{2} \mid \tilde{Z}_{2}\right)
\end{aligned}
$$

where in step (a) we have $V_{1} \sim N\left(0,1-\rho_{2}^{2}\right)$, in step (b) we have $V \sim N\left(0,1-\rho_{2}^{2}-H_{21}^{2} \sigma_{1}^{2}\right)$ and $V$ is independent of $\tilde{Z}_{1}$, and in step (c) we use the fact that removing conditioning that is independent of $V$ does not increase the the mutual information. Note that step (b) holds only when (29) is satisfied. Step (d) follows by the worst case noise lemma [11]. Following the same lines, we can show that if

$$
1-\rho_{1}^{2} \geq H_{12}^{2} \sigma_{2}^{2},
$$

then $U_{1}$ can be bounded in a similar manner. Thus, we have

$$
\begin{aligned}
& T\left(R_{\Sigma}-\epsilon\right) \\
& \leq \quad T h\left(H_{21} X_{1 G}+H_{21} \tilde{Z}_{1}^{(T)}\right)-T h\left(H_{21} X_{1 G}+Z_{2} \mid \tilde{Z}_{2}\right) \\
& \quad+T h\left(H_{12} X_{2 G}+H_{12} \tilde{Z}_{2}\right)-T h\left(H_{12} X_{2 G}+Z_{1} \mid \tilde{Z}_{1}\right) \\
& \quad+T h\left(Y_{1 G} \mid \tilde{S}_{1 G}\right)+T h\left(Y_{2 G} \mid \tilde{S}_{2 G}\right)-T h\left(\tilde{Z}_{1}\right)-T h\left(\tilde{Z}_{2}\right)
\end{aligned}
$$

$$
=T\left[I\left(X_{1 G} ; Y_{1 G}, \tilde{S}_{1 G}\right)+I\left(X_{2 G} ; Y_{2 G}, \tilde{S}_{2 G}\right)\right] .
$$

Dividing both sides by $T$ and letting $T \rightarrow \infty$, we have

$$
R_{\Sigma} \leq I\left(X_{1 G} ; Y_{1 G}, \tilde{S}_{1 G}\right)+I\left(X_{2 G} ; Y_{2 G}, \tilde{S}_{2 G}\right) .
$$

The rest of the proof follows Lemma 10 in [4]. Specifically, it can be shown that if

$$
\begin{aligned}
& H_{11} \sigma_{1} \rho_{1}=H_{12}^{2} P_{2}+1 \\
& H_{22} \sigma_{2} \rho_{2}=H_{21}^{2} P_{1}+1,
\end{aligned}
$$

then

$$
\begin{aligned}
& I\left(X_{1 G} ; Y_{1 G}, \tilde{S}_{1 G}\right)=I\left(X_{1 G} ; Y_{1 G}\right) \\
& I\left(X_{2 G} ; Y_{2 G}, \tilde{S}_{2 G}\right)=I\left(X_{2 G} ; Y_{2 G}\right) .
\end{aligned}
$$

Combining (31), (34), and (35), we get the desire outer bound (20). Also as shown in [4], (19) can be obtained by combining (29), (30), (32), and (33). By letting a genie provide provide $H_{22}\left(X_{2}+\tilde{Z}_{1}\right)$ alone with $W_{22}$ to receiver 1 and $H_{11}\left(X_{1}+\tilde{Z}_{2}\right)$ alone with $W_{11}$ to receiver 2 , we can prove (21) and (22) in a similarly way. This completes the proof.

\section{CONCLUSIONS}

We consider the GDOF and capacity of the two-user Gaussian $X$ channel in this paper. Interesting and potential extention of this work includes finding the complete GDOF characterization of the channel, extending the bounds established in this paper to the case with more users, and approximating the capacity region of the $X$ channel to within a constant number of bits.

\section{REFERENCES}

[1] R. Etkin, D. Tse, and H. Wang, "Gaussian interference channel capacity to within one bit," submitted to IEEE Trans. Inform. Theory, Feb. 2007.

[2] A. Motahari and A. Khandani, "Capacity bounds for the Gaussian interference channel," arXiv:cs/0801.1306, Jan. 2008.

[3] X. Shang, G. Kramer, and B. Chen, "A new outer bound and the noisy-interference sum-rate capacity for gaussian interference channels," submitted to IEEE Trans. Inform. Theory. arXiv:0712.1987, Dec. 2007.

[4] V. Annapureddy and V. Veeravalli, "Gaussian interference networks: Sum capacity in the low interference regime and new outer bounds on the capacity region," Submitted to IEEE Trans. Inform. Theory. arXiv:0802.3495, Feb. 2008.

[5] V. Cadambe and S. Jafar, "Interference alignment and the degrees of freedom of the $\mathrm{K}$ user interference channel," IEEE Trans. Inform. Theory, vol. 54, pp. 3425-3441, Aug. 2008.

[6] L. Zheng and D. Tse, "Diversity and multiplexing : A fundamental tradeoff in multiple-antenna channels," vol. 49, pp. 1073-1096, May 2003.

[7] G. Bresler and D. Tse, "The two-user Gaussian interference channel: A deterministic view," European Trans. Telecommunications, vol. 19, pp. 333-354, Jun. 2008.

[8] S. Jafar and S. Shamai, "Degrees of freedom region for the mimo $X$ channel," IEEE Trans. Inform. Theory, vol. 54, pp. 151-170, Jan. 2008.

[9] M. Maddah-Ali, A. Motahari, and A. Khandani, "Communication over mimo X channels: Interference alignment, decomposition, and performance analysis," IEEE Trans. Inform. Theory, vol. 54, pp. 3457-3470, Aug. 2008.

[10] V. Cadambe and S. Jafar, "Degrees of freedom of wireless X networks," arXiv:0711.2824, Nov 2007.

[11] S. N. Diggavi and T. M. Cover, "The worst additive noise under a covariance constraint," IEEE Trans. Inform. Theory, vol. 47, pp. 30723081, Jul. 2001. 